R. Buta, D. A. Crocker, and B. G. Elmegreen, eds.

\title{
A Gas Dynamical Approach to a Face-on View of Our Galaxy
}

Keiichi Wada

Center for Information Processing Education, Hokkaido University, Sapporo 060, Japan

Yoshiaki Taniguchi

Department of Astronomy, Tohoku Univ., Sendai 980-77, Japan

Asao Habe

Department of Physics., Hokkaido Univ. Sapporo 060, Japan

Tetsuo Hasegawa

Institute of Astronomy, The Univ. of Tokyo, Mitaka, Tokyo 181, Japan

\section{Parameters of the 'Bar' in Our Galaxy}

Recently the bar model for our Galaxy has been an emerging consensus. Here, we investigate how a rotating bar-like bulge affects the global gas dynamics in a disk and compare the longitude-velocity (LV) maps from self-gravitating hydrodynamical simulations, which is based on Wada and Habe (1992), with observed maps of neutral hydrogen and carbon monoxide in the Galaxy. We found that the features on the numerical LV maps depend strongly on four factors: the pattern speed of the bar, the position angle of the Sun, the strength of the bar potential and the ratio of the gas mass to total dynamical mass. We conclude that our Galaxy has a rotating, weak, bar-like bulge $(a / b \sim 0.7)$ observed from nearly end on $\left(\theta_{p}<20^{\circ}\right)$. The allowed range of pattern speed of the bar is surprisingly narrow $\left(19 \pm 5 \mathrm{~km} \mathrm{~s}^{-1} \mathrm{kpc}^{-1}\right)$ and is consistent with recent observations of bulge stars. Self-gravity of the interstellar matter is needed to account for the observed molecular ring at a radius of $\sim 4 \mathrm{kpc}$ even if the gas mass fraction to the dynamical mass is small (about $5 \%$ ).

We can also apply the presented method to the studies of external edge-on galaxies. For example, NGC 891, which is a well-studied nearby edge-on galaxy, also has a central high velocity component and a molecular ring on its observed $\mathrm{HI}$ and CO position-velocity diagram (Sofue \& Nakai 1993). Our study suggests that NGC 891 is quite similar to our Galaxy not only from the edge-on view, but also the face-on view.

\section{Bar Strength and Metal Abundance Gradient}

Our study suggests an important constraint about the strength of the bar. We have tried to reproduce the observed LV diagrams with a more strongly distorted 

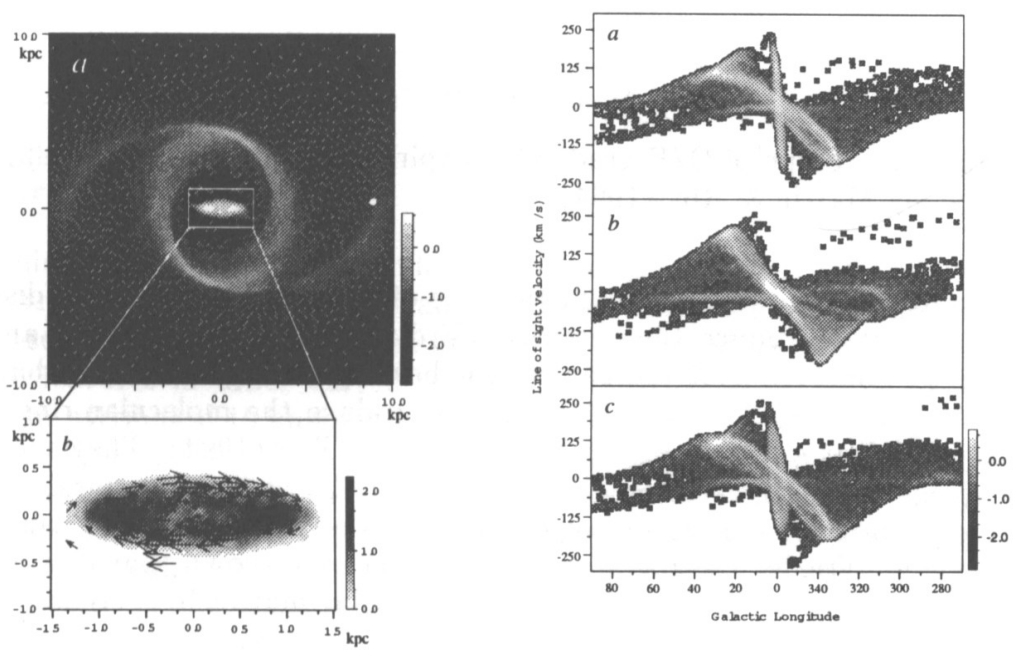

Figure 1. (Left) (a) The most probable model of gas density distribution of our Galaxy. The most probable position of the LSR is shown by a white filled circle. (b) Position-Velocity diagram from the LSR.

Figure 2. (a)(Right) Longitude-velocity diagram for Figure 1(a). The position angle of the LSR, $\theta_{\mathrm{p}}$, is $10^{\circ}$, measured counter-clockwise from the major axis of the bar. The vertical axis is velocity, and the horizontal axis is Galactic longitude. The gray scale represents surface density on the LV plane, $\log _{10}\left(\mu / \mu_{0}\right), \mu_{0} \equiv 3 \times 10^{5} M_{\odot} /\left(\mathrm{km} \mathrm{s}^{-1} \mathrm{deg}\right)$. (b) Same as Figure 2(a), but for $\theta_{\mathrm{p}}=90^{\circ}$. (c) Same as Figure 2(a), but for $\theta_{\mathrm{p}}=30^{\circ}$. 


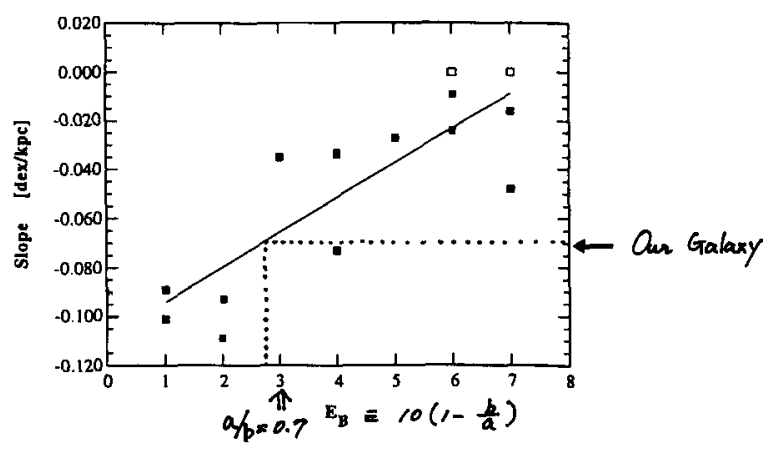

Figure 3. Slope of $\mathrm{O} / \mathrm{H}$ gradients in spiral galaxies vs. the bar ellipticity: by Martin \& Roy (1994)

bar-like potential. However, even in the case of $a / b=0.5$ in the mass distribution, we cannot reproduce the observed LV diagram for any position angle at all. Thus we conclude that the bar cannot be very strong. A weaker bar with $a / b=0.9$ (Model $\mathrm{H})$, however, also fails to produce the molecular ring. This result is consistent wth a recent study by Martin \& Roy (1994). They suggested a very strong correlation between the slope of $0 / \mathrm{H}$ gradients in spiral galaxies and strength of their bar: the stronger the bar, the flatter the abundance gradient becomes. Friedli, Benz \& Kennicutt (1994) have shown that this relation can be explained by redistribution of the interstellar matter by a stellar bar. In our Galaxy, the slope of $\mathrm{O} / \mathrm{H}$ gradients is -0.07 . This value suggests the axial ratio of the bar in our Galaxy is about 0.72 . On the other hand, our prediction is $\sim 0.7$.

We suspect that our Galaxy and NGC 891 are not necessarily 'barred' galaxies but are ordinary spiral galaxies classified as $\mathrm{Sb} \sim \mathrm{Sc}$ in the classification scheme of Hubble. They should look like NGC 309 (Sc) which has a weaklydistorted bar with two open spiral arms when viewed in the infrared (Block \& Wainscoat 1991).

\section{References}

Block, D. L. \& Wainscoat, R. J. 1991, Nature, 353, 48

Friedli, D., Benz, W., \& Kennicutt, R. 1994, ApJ, 430, L105

Martin, P. \& Roy, J.-R. 1994, ApJ, 424, 599

Sofue, Y. \& Nakai, N. 1993, PASJ, 45, 139

Wada, K. \& Habe, A. 1992, MNRAS, 258, 82 